\title{
Daily intake of alfalfa sprouts, but not broccoli sprouts, influence plasma levels of androgen in middle-aged males
}

\author{
Sasaki M', Shinozaki $\mathrm{S}^{1,2^{*}}$, Sasaki $\mathrm{Y}^{3}$, Mabuchi $\mathrm{S}^{1}$, Abe $\mathrm{Y}^{1}$, Eiji Kaneko ${ }^{1}$ and Shimokado K ${ }^{1}$ \\ ${ }^{1}$ Department of Geriatrics and Vascular Medicine, Tokyo Medical and Dental University Graduate School of Medicine, Tokyo, Japan \\ ${ }^{2}$ Department of Arteriosclerosis and Vascular Biology, Tokyo Medical and Dental University Graduate School of Medicine, Tokyo, Japan \\ ${ }^{3}$ Medical Innovation Promotion Center, Institute of Research, Tokyo Medical and Dental University, Tokyo, Japan
}

\begin{abstract}
Objectives: Dihydrotestosterone (DHT) is known as the main cause of androgenetic alopecia (AGA). We previously reported that sulforaphane (SFN), a compound extracted from broccoli, promotes hair regeneration in ob/ob mice by lowering plasma DHT levels. The aim of this study was to assess whether SFN could decrease plasma DHT in human.

Methods: We conducted a randomized, double-blind trial to evaluate the effect of SFN on participants following oral intake of SFN-rich broccoli sprouts compared with alfalfa sprouts (control). Eighty-seven healthy male participants between the ages of 30 and 60 years were divided into two groups: broccoli or alfalfa sprouts daily intake for one month. Serum testosterone and DHT were determined before and after intervention.

Results: Sixty-eight males were enrolled; 34 in the broccoli sprouts group and 34 in the alfalfa sprouts group. Neither testosterone nor DHT levels were decreased in the broccoli sprouts group. While in the alfalfa sprouts group, testosterone, free testosterone and DHT levels were significantly increased. The change in DHT level of the alfalfa sprouts group was significantly greater than in the broccoli group.

Conclusions: We conducted the study to observe the effect of broccoli sprouts on plasma androgen levels, but there was no effect of broccoli sprouts. We showed that oral alfalfa sprouts, but not broccoli sprouts, intake could increase plasma androgen levels in males. Alfalfa sprouts intake can be expected to prevent male late onset hypogonadism (LOH). Further research is needed to reveal whether oral SFN (broccoli sprouts) intake can result in improved AGA.
\end{abstract}

Trial registration: The study registered in the University Hospital Medical Information Network (UMIN) clinical trial report system (identifier: UMIN000022523).

\section{Introduction}

Dihydrotestosterone (DHT) is known as the main cause of androgenetic alopecia (AGA), which is the most common type of hair loss [1-3]. The binding of DHT to androgen receptors is the main processes of androgen-dependent alopecia [3]. DHT shortens the anagen (growth) phase and elongates the telogen (resting) phase of hair growth, resulting in increased immature hair [1] and decreased new hair [4]. We previously reported that sulforaphane (SFN), a compound extracted from broccoli, promotes hair regeneration in $o b / o b$ mice by decreasing plasma DHT levels [5]. To the best of our knowledge, no studies have focused on the effects of SFN on androgen profiles in humans.

DHT is converted from testosterone by $5 \alpha$-reductase, and is degraded to $3 \beta, 17 \beta$-androstanediol ( $3 \beta$-diol) by $3 \alpha$ - hydroxysteroid dehydrogenases ( $3 \alpha-H S D s)$. Currently, $5 \alpha$-reductase inhibitors that suppress the conversion of testosterone to DHT are widely used to treat AGA [6,7]. Lowering DHT levels is expected to produce similar effects as existing AGA therapeutic agents. However, the activation of DHT degrading enzymes and the consequent effects on AGA have not been tested clinically.

Plants contain various substances, termed phytochemicals, which may have beneficial effects on health. SFN is one of the phytochemicals extracted from broccoli [8]. We previously demonstrated that SFN affects plasma androgen levels in $o b / o b$ mice by enhancing DHT- degrading enzymes, such as aldo-keto reductase (AKR) 1c21 and dehydrogenase/reductase (SDR family) member 9 [5]. These enzymes convert DHT to $3 \beta$-diol under reduced (NADPH-rich) conditions [9]. SFN is known to have anti-oxidative effects through activation of the nuclear factor erythroid 2 (NF-E2)-related factor 2 (Nrf2) pathway, including NADPH-producing genes [10,11]. Therefore, SFN promotes the degradation of DHT.

The ultimate goal of this study is to obtain evidence that SFN produces hair regenerative effects in humans. However, since confirmation of such regenerative effects in participants is a long-term undertaking, a first step is to determine whether SFN can decrease plasma DHT. This is a pilot study for the effect of SFN-rich broccoli sprouts on androgen levels in middle-aged males, and was carried out in a randomized and double-blind, and comparison-controlled manner.

${ }^{*}$ Correspondence to: Shohei Shinozaki, Department of Geriatrics and Vascular Medicine, Tokyo Medical and Dental University Graduate School of Medicine, Tokyo, Japan, E-mail: sinozaki.vasc@tmd.ac.jp

Key words: androgenetic alopecia, late onset hypogonadism, dihydrotestosterone, testosterone, sulforaphane, broccoli, alfalfa

Received: October 05, 2018; Accepted: October 12, 2018; Published: October 17 , 2018 


\section{Material and methods}

\section{Study design}

This was a single-center, randomized, double-blind, pilot study comparing the effects of broccoli sprouts to control (alfalfa sprouts) in middle-aged healthy men. The study was conducted at Tokyo Medical and Dental University in Tokyo, Japan. The study was approved by the Committee on Clinical Investigation at Tokyo Medical and Dental University (approval number: M2016-047) and participants provided written informed consent prior to enrollment. The trial was registered in the University Hospital Medical Information Network (UMIN) clinical trial report system (identifier: UMIN000022523) and was sponsored by Murakami Farm Corporation, Japan. The trial was investigator-initiated and the sponsor was not involved in the study design or conduct, and had no role in manuscript preparation.

\section{Participants}

Volunteer adults were recruited by the CX Medical Japan Co., Ltd. Eligibility criteria were healthy males between 30 and 60 years of age who did not take supplemental vitamins or mineral pills. Participants did not consume any topical or oral medication such as minoxidil, dutasteride, finasteride, steroids or supplements that may affect androgen profiles before the study. Participants were excluded if they had serious diseases such as diabetes and cancer, or if they had an allergy against broccoli or alfalfa sprouts. Randomization was performed at a 1:1 ratio using number cards. The study treatment was randomly assigned and labeled with the participant numbers before initiation of the study. A total of 86 participants were randomly assigned to either of the two study groups: broccoli or alfalfa sprouts daily intake for one month.

\section{Intervention}

We established the starting dose of SFN in humans using the formula of translation from animal to human equivalent dose [12]. Based on this formula and our previous study [5], about $0.36 \mathrm{mg} /$ $\mathrm{kg}$ body weight per day SFN is required. The daily intake of broccoli sprouts based on the required dose of SFN was determined in reference to the following two papers. Broccoli super sprouts ${ }^{\star}$ contain $6 \mu \mathrm{mol} / \mathrm{g}$ SFN [13], and the bioavailability of SFN from broccoli sprouts is about $60 \%$ [14]. Therefore, we calculated that about $50 \mathrm{~g}$ per day broccoli sprouts was required for this study. We chose alfalfa sprouts as the control because the total amounts of genistein and daizein (isoflavones) content is same as broccoli sprouts, but alfalfa sprouts do not contain significant amounts of SFN [15]. In order to resemble the appearance of broccoli sprouts, alfalfa sprouts were irradiated with sunlight, and the color and form was made the same as broccoli sprouts. Comparison group was given $50 \mathrm{~g}$ per day alfalfa in other reports of human subjects in comparison with broccoli sprouts [16-18]. Participants did not know which sprouts they were taking daily.

Broccoli and alfalfa sprouts industrially produced by Murakami Farm Corporation were used in the present study. Broccoli and alfalfa sprouts were grown from specially selected seeds for 2 days following germination. The sprouts were then provided to participants twice a week so that participants could consume them within 5 days of germination. Participants were instructed to consume one pack (50 g) daily for 4 weeks with meals. They were also asked to maintain their regular diet and lifestyle, during the study period. Participants were required to record their compliance to sprouts intake and general health condition. Based on the participants' compliance to sprouts intake, if adverse reactions such as gastrointestinal events (including diarrhea and vomiting) were observed, the trial was stopped immediately.

\section{Biochemical analysis}

At baseline and 4 weeks after intervention, $12 \mathrm{~h}$ fasting blood samples were collected into tubes with or without EDTA before noon. Samples were centrifuged at $4^{\circ} \mathrm{C}$ and $1,000 \mathrm{x}$ g for $15 \mathrm{~min}$ to separate plasma or serum. Plasma total testosterone was measured with solid phase radioimmunoassay, free testosterone was measured with a chemiluminescent immunoassay (BML, Tokyo, Japan), and DHT was measured with a Dihydrotestosterone ELISA KIT (Alpha Diagnostics, San Antonio, TX). DHT level was re-measured using a diluted sample when the result was beyond the detection limit of the kit. However, some samples showed extremely high DHT levels with this ELISA kit because of hemolysis; thus, we re-confirmed the high DHT level samples above 2,500 $\mathrm{pg} / \mathrm{ml}$ (and also some normal samples for assay confirmation) using radio-immunoassay conducted by LSI Medience (Tokyo, Japan). We excluded hemolyzed samples from the assay. At baseline, fasting blood glucose, white blood cell count, red blood cell count, hematocrit, total protein, albumin, aspartate aminotransferase (AST), alanine aminotransferase (ALT), lactate dehydrogenase (LDH), alkali phosphatase (ALP), and creatinine were automatically measured using an Auto Blood Biochemistry Analyzer (BML).

\section{Statistical analysis}

The trial was designed for an enrollment of 80 participants ( 40 per group) in order to achieve $80 \%$ power to detect a $25 \%$ change in plasma DHT level with a two-sided type 1 error rate of 0.05 to account for comparisons. Standard deviation of the plasma DHT level was calculated as $230 \mathrm{pg} / \mathrm{ml}$ based on assessments in Japanese males for dutasteride [19]. Statistical analysis was performed using JMP, version 13 (SAS Institute Inc., Cary, NC). Data are presented as the mean \pm SEM or median \pm quartiles, depending on the normality of the data. A p-value $<0.05$ was considered statistically significant.

\section{Results}

\section{General participant characteristics}

A total of 86 participants were randomized into the 2 study groups. Of those, 79 participants completed the study (broccoli sprouts group, $\mathrm{n}=40$; alfalfa sprouts group, $\mathrm{n}=39$ ) (Figure 1). Broccoli and alfalfa sprouts were well tolerated and the participants reported no serious adverse reactions. In the broccoli sprouts group, minor side effects including gastrointestinal disorder $(n=3)$ were reported. A total of 9 participants were excluded post-randomization because of minor side effects $(n=3)$, fever $(n=1)$, or hemolysis $(n=5)$. In the alfalfa sprouts group, gastrointestinal disorder $(n=3)$ was reported. There were no significant differences between the baseline characteristics of the broccoli sprouts group $(n=34)$ and the alfalfa sprouts group $(n=34)$ (Table 1).

\section{Plasma androgen levels}

Testosterone, free testosterone and DHT levels at baseline and post intervention are shown in Table 2. Plasma concentration of DHT measured with an ELISA kit included some abnormal values. Hence, we re-analyzed the samples that showed abnormal values using another method (radio-immunoassay). These samples showed reasonable values (within the normal range; $200-1000 \mathrm{pg} / \mathrm{ml}$ ). Therefore, we excluded 10 participants (broccoli sprouts group, $n=5$; alfalfa sprouts group, $n=5$ ) from each group because of the limitations of the ELISA assay. The broccoli sprouts group did not show a significant reduction in androgen levels. On the other hand, the alfalfa sprouts group showed a significant increase in androgen (Figure 2). The change in DHT level 


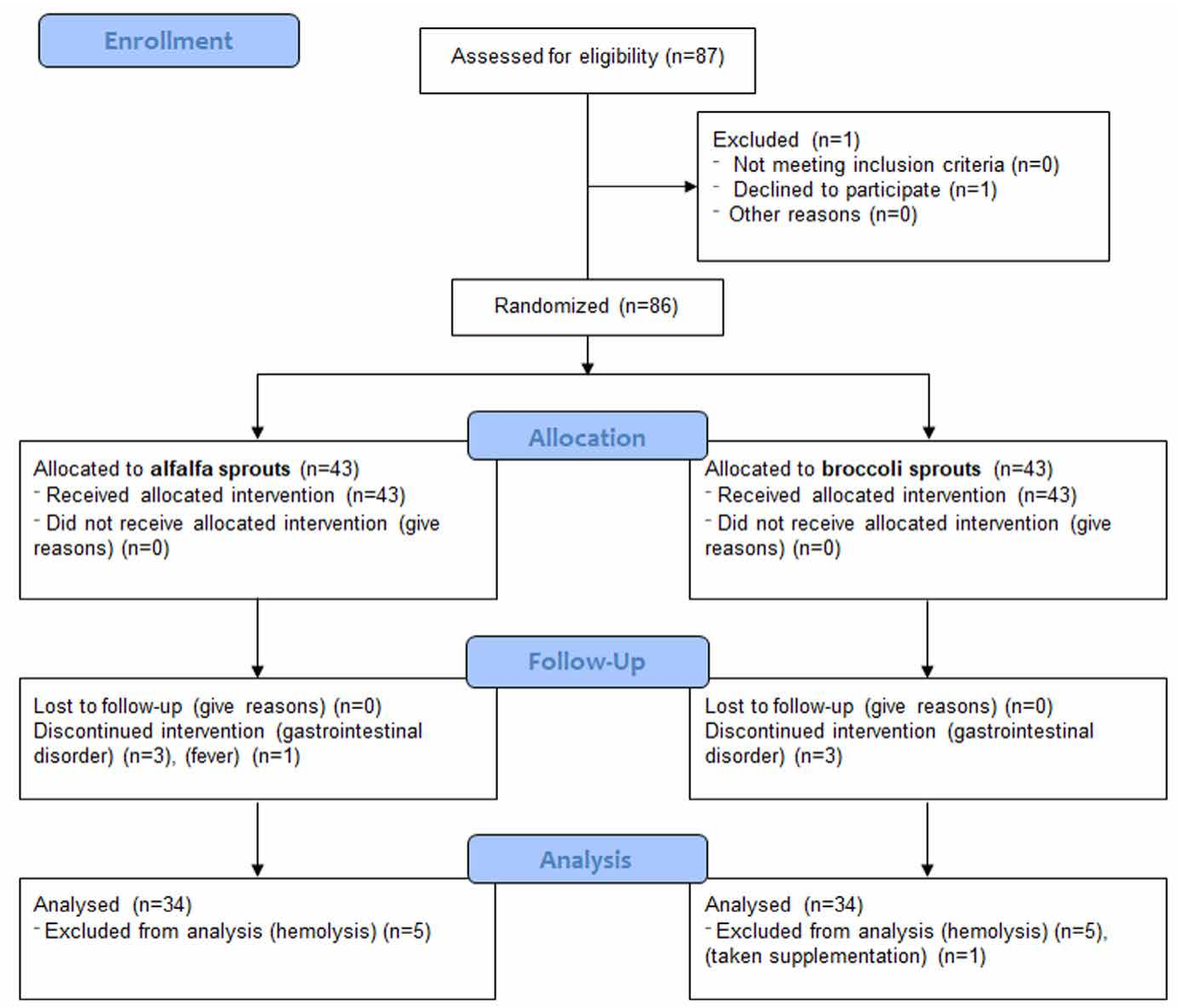

Figure 1. Consort 2010 flow diagram for recruitment and randomization of participants

Table 1. Baseline data of study groups. There were no differences between the alfalfa and broccoli sprouts groups. Values are expressed as the mean \pm SEM. The differences in each parameter were evaluated by a two-tailed, non-paired Student's t-test

\begin{tabular}{|c|c|c|}
\hline & Alfalfa sprouts group $(n=34)$ & Broccoli sprouts group $(n=34)$ \\
\hline Body Weight (kg) & $77.8 \pm 12.2$ & $71.4 \pm 10.4$ \\
\hline Height $(\mathrm{cm})$ & $173.3 \pm 6.7$ & $171.0 \pm 6.6$ \\
\hline BMI $\left(\mathrm{kg} / \mathrm{m}^{2}\right)$ & $24.9 \pm 3.9$ & $24.4 \pm 3.4$ \\
\hline Age (years) & $47.6 \pm 8.1$ & $48.5 \pm 9.7$ \\
\hline White blood count (counts $/ \mu \mathrm{l}$ ) & $5764.0 \pm 1346.7$ & $5597.9 \pm 1313.5$ \\
\hline Red blood count (counts/ $\mu \mathrm{l}$ ) & $487.6 \pm 41.1$ & $493.0 \pm 42.5$ \\
\hline Hematocrit (\%) & $47.4 \pm 3.0$ & $48.0 \pm 3.0$ \\
\hline Total protein $(\mathrm{mg} / \mathrm{dl})$ & $7.3 \pm 0.4$ & $7.3 \pm 0.44$ \\
\hline Albumin (mg/dl) & $4.4 \pm 0.2$ & $4.4 \pm 0.3$ \\
\hline AST (IU/L) & $28.0 \pm 11.5$ & $29.3 \pm 28.1$ \\
\hline ALT (IU/L) & $33.5 \pm 18.8$ & $32.6 \pm 29.4$ \\
\hline LDH (IU/L) & $190.8 \pm 30.3$ & $193.3 \pm 39.4$ \\
\hline ALP (IU/L) & $212.3 \pm 45.4$ & $206.1 \pm 56.5$ \\
\hline Creatinine (mg/dl) & $0.81 \pm 0.10$ & $0.83 \pm 0.10$ \\
\hline
\end{tabular}

Table 2. The effect of alfalfa sprouts intake on plasma androgen levels. Plasma androgen levels were analyzed before and after intervention. There were significant differences between before and after intervention with alfalfa sprouts intake. There were no differences between alfalfa and broccoli sprouts groups in plasma testosterone and free testosterone. $\mathrm{N}=34$ participants per group. ${ }^{*} \mathrm{p}<0.05,{ }^{*} \mathrm{p}<0.01 \mathrm{vs}$. baseline

\begin{tabular}{|c|c|c|c|c|}
\hline & \multicolumn{2}{|c|}{ Alfalfa sprouts group (n=34) } & \multicolumn{2}{c|}{ Broccoli sprouts group (n=34) } \\
\hline & Before & After & After \\
\hline Testosterone $(\mathrm{pg} / \mathrm{ml})$ & $587.9(439.9-703.7)$ & $583.3(499.3-759.2)^{*}$ & $592.0(417.6-735.0)$ \\
\hline Free testosterone $(\mathrm{pg} / \mathrm{ml})$ & $10.2(8.4-12.5)$ & $11.8(9.7-12.7)^{*}$ & $10.6(8.6-12.9)$ \\
\hline DHT $(\mathrm{pg} / \mathrm{ml})$ & $535.6(455.3-726.0)$ & $662.3(547.7-761.7)^{* *}$ & $557.4(471.6-734.4)$ \\
\hline
\end{tabular}

*Values with continuous values are expressed as median (interquartile range). Differences in each parameter of the same group was evaluated by a two-tailed, Wilcoxon signed-rank test and between groups were evaluated by a two-tailed, Mann-Whitney U test 
of the alfalfa sprouts group was significantly greater than in the broccoli group (Figure 3). However, there were no significant differences in testosterone and free testosterone between the groups (Figure 3).

\section{Discussion}

In this study, we demonstrated that the daily intake of alfalfa sprouts, but not broccoli sprouts, influences androgen levels in human male participants. To the best of our knowledge, this is the first report to demonstrate that alfalfa sprouts have an androgen-inducing effect in humans. In this study, broccoli sprouts were not observed to have a DHT-reducing effect. In addition, there were no severe side effects or allergic reactions such as rash.

We expected that broccoli sprouts would have a DHT-reducing effect in humans. In contrast to our previous report [5], we could not demonstrate the effect of SFN on androgen profiles in human male subjects. There are some possible reasons why we did not observe a plasma androgen-decrease effect of SFN in human subjects. First, the method of SFN treatment differed from the previous report; mice were treated with an intraperitoneal injection of SFN, whereas human subjects obtained SFN by oral intake of broccoli sprouts. We estimated the intake dose of broccoli sprouts (SFN) in humans using a formula for determining human equivalent dose and previous studies [12-14]. However, individual variation in chewing, time of intake and ingestive function may have influenced the results. In addition, purified SFN alone was not given to human subjects; therefore, we could not rule out the possibility that other hormone-like components such as isoflavones (e.g., genistein, daizein and quercetin) masked the effects of SFN. Secondly, the number of participants was not sufficient to demonstrate a significant change in androgen levels, since androgen levels varied greatly among the participants, and the estimated change ratio (25\%) was too large to detect significant differences. It is important to note
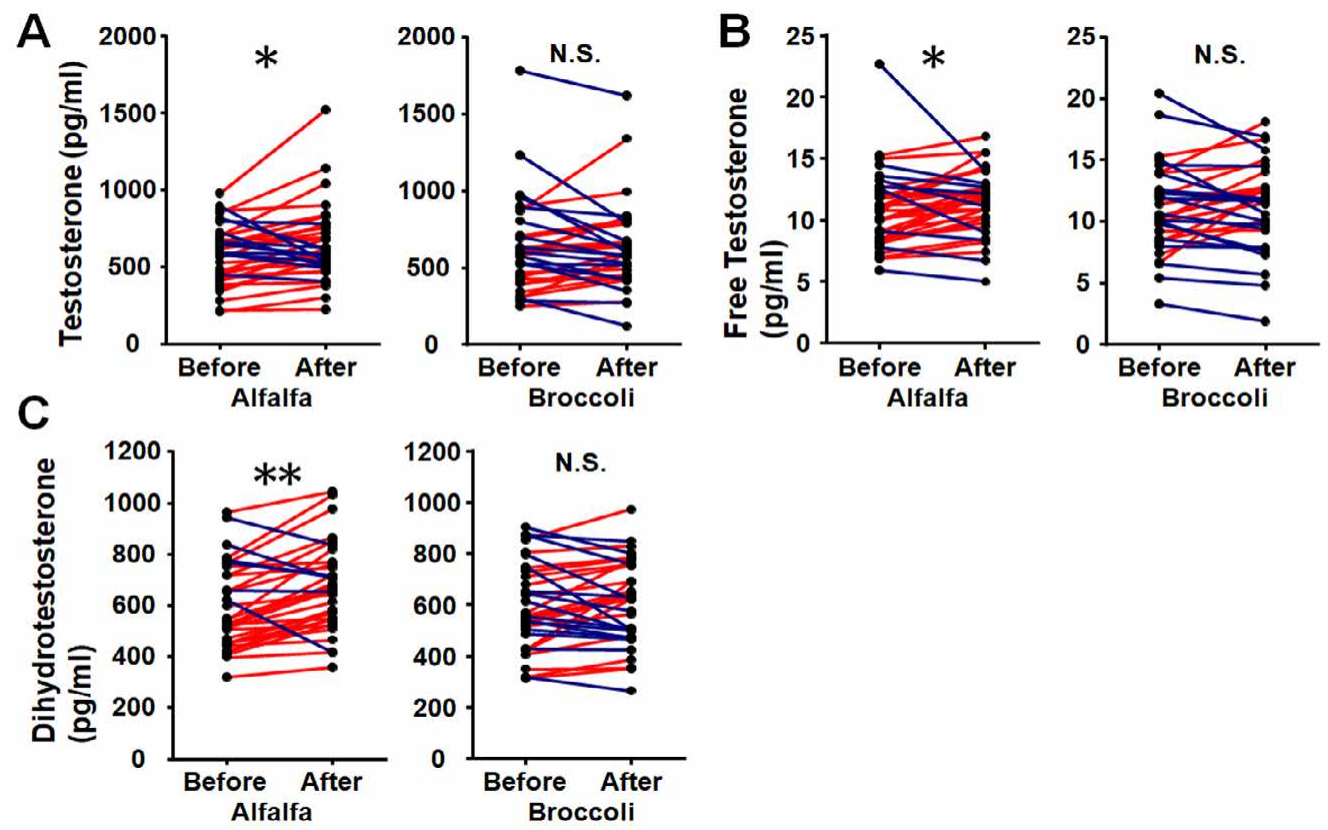

Figure 2. The effect of broccoli sprouts intake on plasma androgen levels. Plasma testosterone (A), free testosterone (B) and dihydrotestosterone (C) were analyzed before and after intervention. There were significant differences with alfalfa intake on androgen levels compared to before intervention. No differences were observed with broccoli sprouts intake. The red lines indicate increased, the blue lines indicate decreased compare with baseline (before). $\mathrm{N}=34$ participants per group. ${ }^{*} \mathrm{p}<0.05,{ }^{* *} \mathrm{p}<0.01$ vs. baseline. N.S. not significant. The differences in androgen levels were evaluated by a two-tailed, Wilcoxon signed-rank test

A

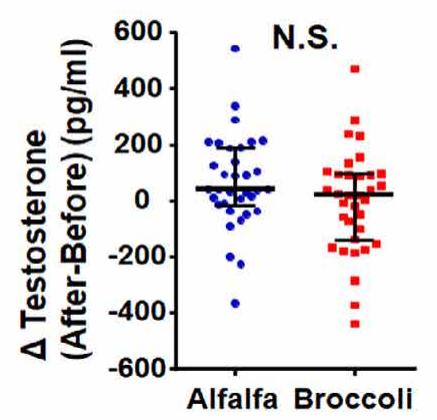

B

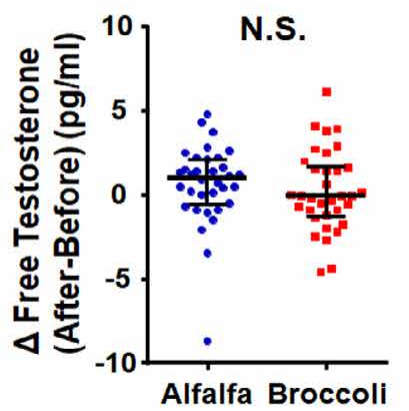

C

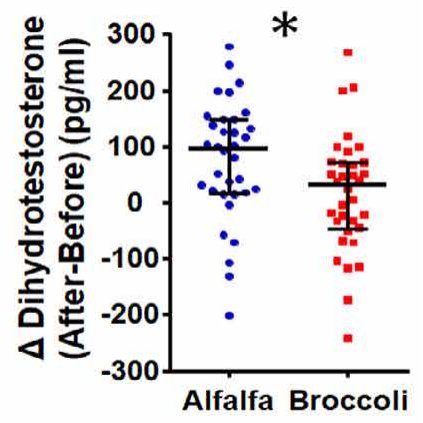

Figure 3. The effect of alfalfa sprouts intake on plasma dihydrotestosterone levels. Changes in levels of plasma testosterone (A), free testosterone (B) and dihydrotestosterone (C) were analyzed between the alfalfa and broccoli sprouts groups. A significant difference in dihydrotestosterone levels was observed with alfalfa intake. There were no differences for plasma testosterone and free testosterone levels. *Values showing median and error bars indicate the interquartile range. $\mathrm{N}=34$ participants per group. ${ }^{*} \mathrm{p}<0.05$, vs. broccoli sprouts group. N.S. not significant. Differences in androgen levels were evaluated by a two-tailed, Mann-Whitney U test 
that the genetic background of human subjects varies to a larger extent relative to the genetically homogenous mice strain, and the effect of SFN may have not produced the same effect in humans. Thirdly, we could not exclude the influence of alcohol. Based on our hypothesis, SFN induces $3 a-H S D$ s in the liver, and these enzymes subsequently decompose DHT. These enzymes are also induced by alcohol via the Nrf2 pathway [20]; therefore, participants who habitually drink alcohol might have limited ability to induce these enzymes. In fact, plasma levels of DHT tended to decrease with broccoli sprouts intake, with the exception of participants who appeared to show alcohol-associated impacts on liver function $(\gamma-\mathrm{GTP}<50 \mathrm{IU} / \mathrm{L}$ and ALP $<260 \mathrm{IU} / \mathrm{L})(\mathrm{S} 1$ Table).

In this study, we did not observe a reduction in DHT with oral intake of broccoli sprouts. Similarly, we were not able to confirm the effect of hair regeneration by oral intake of broccoli sprouts. It has been reported that SFN also activates Nrf2 in the skin (mice keratinocytes) [21]. Therefore, a hair growth promoting effect can be expected even if systemic DHT concentrations are not decreased. Notably, the human life span (as well as the hair cycle) is longer than that of mice. Thus, long-term observations are needed to elucidate whether SFN has a hair growth promoting effect via the oral intake of broccoli sprouts.

We also showed an unexpected effect of alfalfa sprouts on plasma androgen levels. Free testosterone is known to decrease with age, and is one of the indicators of male late onset hypogonadism (LOH) [19]. The intake of alfalfa sprouts increased the $70 \%$ average value of free testosterone of participants in their $40-50$ s to the $70 \%$ average value seen in 20-30 year olds [19] (Table 2). Thus, alfalfa sprouts intake can be expected to prevent LOH. Alfalfa is known to contain phytoestrogens, such as genistein and daizein, which act as agonists of estrogen receptors [15,22]. Broccoli sprouts also contain daizein; however, the total amount of both isoflavones (genistein and daizein) was the same in both sprouts [15]. Therefore, we suggest that the effects of isoflavones could be ignored within both groups when comparing the effects of SFN. On the other hand, both sprouts contain quercetin, a one of isoflavone [22,23]. There are conflicting reports that quercetin has anti- and/or estrogen-like effects [24-28]. Quercetin has been reported to inhibit testosterone-mefigureolizing enzymes and to increase blood testosterone concentration [27]. Moreover, quercetin has been reported to increase testosterone production in rat Leydig cells [28]. Therefore, the blood androgen increasing effect of alfalfa is possibly due to quercetin. We have thought that an increase in androgens was not observed in the broccoli sprouts group because the effect of SFN was negated by the effect of quercetin.

We believe that this is the first report to suggest that the oral intake of alfalfa sprouts may contribute to increasing androgen levels in middle-aged males. Further research on the mechanism of this effect as well as human health safety implications is needed.

\section{Conclusions}

In this study, we demonstrated that the daily intake of alfalfa sprouts, but not broccoli sprouts, increased androgen levels in human male subjects. Alfalfa sprouts intake can be expected to prevent $\mathrm{LOH}$, however, further research on the mechanism of effect of alfalfa as well as human health safety implications is needed.

\section{Acknowledgements}

We thank Ms. M. Harada for her valuable technical assistance. This study was sponsored by Murakami Farm Corporation. This study was supported by grant from JSPS KAKENHI (18K06952) to SS.

\section{Author contributions}

Conceived and designed the experiments: MS, SS, KS. Performed the experiments: MS, SM, YA, EK. Analyzed the data: MS, YS, SS. Wrote the manuscript: MS, SS, KS.

\section{References}

1. Hoffmann R (2002) Hormonal interaction and hair growth. Ann Dermatol Venereol 129: 787-792. [Crossref]

2. Prager N, Bickett K, French N, Marcovici G (2002) A randomized, double-blind, placebo-controlled trial to determine the effectiveness of botanically derived inhibitors of 5-alpha-reductase in the treatment of androgenetic alopecia, Journal of alternative and complementary medicine 8: 143-152. [Crossref]

3. Trüeb RM (2002) Molecular mechanisms of androgenetic alopecia Experimental gerontology 37: 981-990. [Crossref]

4. Olsen EA, Hordinsky M, Whiting D, Stough D, Hobbs S, et al. (2006) The importance of dual 5alpha-reductase inhibition in the treatment of male pattern hair loss: results of a randomized placebo-controlled study of dutasteride versus finasteride $J \mathrm{Am} \mathrm{Acad}$ Dermatol 55: 1014-1023. [Crossref]

5. Sasaki M, Shinozaki S, Shimokado K (2016) Sulforaphane promotes murine hair growth by accelerating the degradation of dihydrotestosterone. Biochem Biophys Res Commun 472: 250-254. [Crossref]

6. Gupta AK, Charrette A (2014) The efficacy and safety of 5alpha-reductase inhibitors in androgenetic alopecia: a network meta-analysis and benefit-risk assessment of finasteride and dutasteride, J Dermatolog Treat 25: 156-161. [Crossref]

7. Varothai S, Bergfeld WF (2014) Androgenetic alopecia: an evidence-based treatment update. Am J Clin Dermatol 15: 217-230. [Crossref]

8. Zhang Y, Talalay P, Cho CG, Posner GH (1992) A major inducer of anticarcinogenic protective enzymes from broccoli: isolation and elucidation of structure. Proc Natl Acad Sci U S A 89: 2399-2403. [Crossref]

9. Chetyrkin SV, Belyaeva OV, Gough WH, Kedishvili NY (2001) Characterization of a novel type of human microsomal 3alpha -hydroxysteroid dehydrogenase: unique tissue distribution and catalytic properties. The Journal of biological chemistry 276: 2227822286

10. Prawan A, Keum YS, Khor TO, Yu S, Nair S (2008) Structural influence of isothiocyanates on the antioxidant response element (ARE)-mediated heme oxygenase-1 (HO-1) expression, Pharmaceutical research 25: 836-844. [Crossref]

11. Sun CC, Li SJ, Yang CL, Xue RL, Xi YY (2015) Sulforaphane attenuates muscle inflammation in dystrophin-deficient mdx Mice via NF-E2-related Factor 2 (Nrf2)mediated Inhibition of NF-kappaB Signaling Pathway. The Journal of biological chemistry 290: 17784-17795. [Crossref]

12. Food D (2005) Administration guidance for industry: estimating the maximum safe starting dose in initial clinical trials for therapeutics in adult healthy volunteers. Centre for drug evaluation and research 7.

13. Yanaka (2011) Sulforaphane enhances protection and repair of gastric mucosa agains oxidative stress in vitro, and demonstrates anti-inflammatory effects on Helicobacter pyloriinfected gastric mucosae in mice and human subjects. Current pharmaceutical design 17: 1532-1540. [Crossref]

14. Fahey JW, Holtzclaw WD, Wehage SL, Wade KL, Stephenson KK, et al. (2015) Sulforaphane bioavailability from glucoraphanin-rich broccoli: Control by active endogenous myrosinase. PloS one 10: e140963. [Crossref]

15. Bhagwat S, Gebhardt S, Haytowitz D, Holden J, Harnly J (2007) USDA database for the flavonoid content of selected foods: release 2.1, Washington, DC: US Department of Agriculture.

16. Chen YJ, Wallig MA, Jeffery EH (2016) Dietary broccoli lessens development of fatty liver and liver cancer in mice given diethylnitrosamine and fed a western or control diet-3. The Journal of nutrition 146: 542-550. [Crossref]

17. Hanlon N, Coldham N, Gielbert A, Sauer MJ (2009) Repeated intake of broccoli does not lead to higher plasma levels of sulforaphane in human volunteers. Cancer letters 284: $15-20$.

18. Yanaka A, Fahey JW, Fukumoto A, Nakayama M, Inoue S (2009) Dietary sulforaphanerich broccoli sprouts reduce colonization and attenuate gastritis in Helicobacter pyloriinfected mice and humans, Cancer Prevention Research 2: 353-360. 
19. Tsukamoto T, Endo Y, Narita M (2009) [Assessment of recommended dose of dutasteride on Japanese men with benign prostatic hyperplasia: a randomized, doubleblind, placebo-controlled, parallel-group, dose response study]. Hinyokika Kiyo 55: 209-214.

20. O'connor T, Ireland LS, Harrison DJ, Hayes JD (1999) Major differences exist in the function and tissue-specific expression of human aflatoxin B1 aldehyde reductase and the principal human aldo-keto reductase AKR1 family members. The Biochemical journal 343: 487-504. [Crossref]

21. Kleszczyński K, Ernst IM, Wagner AE, Kruse N, Zillikens D, et al. (2013) Sulforaphane and phenylethyl isothiocyanate protect human skin against UVR-induced oxidative stress and apoptosis: role of Nrf2-dependent gene expression and antioxidant enzymes. Pharmacological research 78: 28-40. [Crossref]

22. Bora KS, Sharma A (2011) Phytochemical and pharmacological potential of Medicago sativa: a review. Pharmaceutical biology 49: 211-220. [Crossref]

23. Ferruzza S, Natella F, Ranaldi G, Murgia C, Rossi C, et al. (2016) Nutraceutical improvement increases the protective activity of broccoli sprout juice in a human intestinal cell model of gut inflammation. Pharmaceuticals 9: 48. [Crossref]
24. Pang XG, Cong Y, Bao NR, Li YG, Zhao JN (2018) Quercetin stimulates bone marrow mesenchymal stem cell differentiation through an estrogen receptor-mediated pathway. BioMed research international 2018: 4178021.

25. Shahzad H, Giribabu N, Muniandy S, Salleh N (2014) Quercetin induces morphological and proliferative changes of rat's uteri under estrogen and progesterone influences. Int J Clin Exp Pathol 7: 5484-5494. [Crossref]

26. Yang JX, Chaudhry MT, Yao JY, Wang SN, Zhou B, et al. (2018) Effects of phytooestrogen quercetin on productive performance, hormones, reproductive organs and apoptotic genes in laying hens. J Anim Physiol Anim Nutr 102: 505-513. [Crossref]

27. Zielinski J, Mevissen M (2015) Inhibition of in vitro metabolism of testosterone in human, dog and horse liver microsomes to investigate species differences. Toxicol In Vitro 29: 468-478. [Crossref]

28. Cormier M, Ghouili F, Roumaud P, Martin LJ, Touaibia M (2017) Influence of flavonols and quercetin derivative compounds on MA-10 Leydig cells steroidogenic genes expressions. Toxicol In Vitro 44: 111-121. [Crossref]

Copyright: ( 2018 Sasaki M. This is an open-access article distributed under the terms of the Creative Commons Attribution License, which permits unrestricted use, distribution, and reproduction in any medium, provided the original author and source are credited. 